\title{
Comparison of Travoprost and Bimatoprost plus Timolol Fixed Combinations in Open-Angle Glaucoma Patients Previously Treated with Latanoprost plus Timolol Fixed Combination
}

\author{
MARCO CENTOFANTI, FRANCESCO ODDONE, STEFANO GANDOLFI, ANTON HOMMER, \\ ANDREAS BOEHM, LUCIA TANGA, CHIARA SANGERMANI, VITO SPORTELLI, MICHAEL HAUSTEIN, \\ GIANLUCA MANNI, AND LUCA ROSSETTI
}

- PURPOSE: To compare the ocular hypotensive effect of bimatoprost plus timolol and travoprost plus timolol fixed combinations in glaucoma patients whose disease was controlled but had not reached their target intraocular pressure (IOP) with the fixed combination of latanoprost plus timolol.

- DESIGN: A 2 × 3-month, multicenter, prospective, randomized, double-masked, cross-over clinical trial.

- METHODS: Eighty-nine open-angle glaucoma (OAG) patients were included. After a 6-week run-in period with latanoprost plus timolol, patients were randomized to either travoprost plus timolol or bimatoprost plus timolol for 3 months. Patients then switched to the opposite therapy for 3 additional months. The primary end point was the comparison of mean daily IOP after 3 months of each treatment.

- RESUlts: At baseline, mean IOP was $16.5 \mathrm{~mm} \mathrm{Hg}$ (95\% confidence interval, 16.0 to $17.0 \mathrm{~mm} \mathrm{Hg}$ ) with treatment with latanoprost plus timolol. Both bimatoprost plus timolol and travoprost plus timolol statistically significantly reduced the mean IOP from baseline $(\mathrm{P}<$ $.0001)$. Mean IOP at month 3 was statistically significantly lower in the bimatoprost plus timolol group compared with the travoprost plus timolol group $(14.7 \mathrm{~mm}$ $\mathrm{Hg}$ [95\% confidence interval, 14.3 to $15.3 \mathrm{~mm} \mathrm{Hg}$ ] vs $15.4 \mathrm{~mm} \mathrm{Hg}$ [95\% confidence interval, 15.0 to $15.9 \mathrm{~mm}$ $\mathrm{Hg}$ ]; $\mathrm{P}=.0041$ ). IOP was lower during bimatoprost plus timolol treatment at all time points and statistical significance was reached at $8 \mathrm{AM}, 11 \mathrm{AM}$, and $5 \mathrm{PM}$, but not at $2 \mathrm{PM}$ and $8 \mathrm{PM}$. Both treatments showed similar tolerability profile.

\section{Accepted for publication May 5, 2010.}

From the Unità Dipartimentale Semplice di Glaucoma, University of Tor Vergata, Rome, Italy (C.M., M.G.); the Istituto di Ricovero e Cura a Carattere Scientifico Fondazione G.B. Bietti, Rome, Italy (C.M., O.F., T.L.); the Institute of Ophthalmology, University Hospital of Parma, Parma, Italy (G.S., S.C.); Hera Hospital, Vienna, Austria (H.A.); the Department of Ophthalmology, University of Dresden, Dresden, Germany (B.A., H.M.); the Augenklinik Elblandklinikum Radebeul, Radebeul, Germany (B.A.); and the Clinica Oculistica, Dipartimento di Medicina, Chirurgia e Odontoiatria, University of Milan, San Paolo Hospital, Milan, Italy (S.V., R.L.).

Inquiries to Marco Centofanti, Via Livenza 3, 00198 Rome, Italy; e-mail: marco.centofanti@fastwebnet.it
- CONCLUSIONS: Bimatoprost plus timolol and travoprost plus timolol can provide additional IOP-lowering effect in patients not fully controlled with latanoprost plus timolol. The observed additional IOP reduction was greater with bimatoprost plus timolol with a similar tolerability profile. (Am J Ophthalmol 2010;150: 575-580. (C) 2010 by Elsevier Inc. All rights reserved.)

OWERING INTRAOCULAR PRESSURE (IOP) IS THE only evidence-based method for treating glaucoma ${ }^{1-3}$ and has been shown to reduce risk of visual field progression from $13 \%$ to $19 \%$ per $1 \mathrm{~mm} \mathrm{Hg}$ of IOP lowering. ${ }^{2-4}$ According to the European Glaucoma Society Guidelines, topical monotherapy is the first step in the medical management ${ }^{5}$ and prostaglandin analogs are the most widely used ocular hypotensive agents for their IOP-lowering efficacy and safety profile, ${ }^{6,7}$ and if target pressure is not reached, it is recommended to switch drugs or to add another drug.

The use of combination therapy frequently is necessary at any stage of the disease, ${ }^{8,9}$ as it has been reported in the Ocular Hypertension Treatment Study ${ }^{9}$ and in the Collaborative Initial Glaucoma Treatment Study, ${ }^{10}$ where up to $50 \%$ and $75 \%$ of patients, respectively, required 2 or more drugs to reach their target pressure. When 2 drugs are required to control the $\mathrm{IOP}$, there are a number of potential advantages in using a fixed combination rather than separate drugs, including no risk of drug washout, ${ }^{11}$ reduced exposure to preservatives, and ultimately better patient compliance and quality of life. ${ }^{12}$ The first prostaglandin fixed combination available in the market was latanoprost plus timolol followed by the fixed combination of travoprost plus timolol and bimatoprost plus timolol. Although there are several reports comparing the hypotensive effect and tolerability of the different prostaglandin analog monotherapies, ${ }^{13}$ fewer studies are available comparing prostaglandin analog fixed combinations. ${ }^{14,15}$ The purpose of this study was to compare the ocular hypotensive effect of bimatoprost plus timolol and travoprost plus timolol in glaucoma patients not fully controlled with latanoprost plus timolol. 


\section{METHODS}

THIS 6-MONTH PROSPECTIVE, MULTICENTER, RANDOMIZED, double-masked, crossover clinical study was carried out at 5 European centers. Patients of 18 years or older who fulfilled the eligibility requirements detailed below and signed an informed consent at the screening visit were included.

- INCLUSION CRITERIA: Inclusion criteria included diagnosis of primary open-angle glaucoma based on the European Glaucoma Society Guidelines criteria, ${ }^{5}$ treatment with nonfixed combination of latanoprost and timolol for at least 3 months or latanoprost plus timolol fixed combination for at least 6 months, IOP less than $21 \mathrm{~mm}$ $\mathrm{Hg}$ at the baseline $8 \mathrm{Am}$ time point on latanoprost plus timolol fixed combination treatment, and target IOP not reached as set by the treating physician.

- EXCLUSION CRITERIA: Exclusion criteria included contraindications to $\beta$-blockers; closed or barely open anterior chamber angles or a history of acute angle closure; ocular surgery or argon laser trabeculoplasty within the previous 3 months; ocular inflammation or infection occurring within 3 months before the pretrial visit; neovascular patients (including diabetic patients with neovascular glaucoma); concomitant topical ocular medications that can interfere with study medications; hypersensitivity to benzalkonium chloride or to any other component of the trial drug solutions; other abnormal condition or symptom preventing the patient from entering the trial, according to the investigator's judgment; any history of refractive surgery, pregnancy, breastfeeding, or childbearing potential without adequate contraception; inability to adhere to the treatment and visit plan; and participation in any other clinical trials within 3 months before the pretrial visit.

- STUDY PLAN: Patients treated with latanoprost plus timolol with an IOP of less than $21 \mathrm{~mm} \mathrm{Hg}$ but requiring further IOP reduction were enrolled in the study. Patients receiving the nonfixed combination were checked for eligibility and scheduled for a wash-in period of 6 weeks with latanoprost plus timolol fixed combination at the prescreening visit.

At the baseline visit, patients were randomized to either bimatoprost plus timolol or travoprost plus timolol and were treated for 3 months. After 3 months, the patients randomized to bimatoprost plus timolol were switched to travoprost plus timolol and patients randomized to travoprost plus timolol were switched to bimatoprost plus timolol. There was no wash-out between the 2 treatments.

Assessment of baseline data was conducted before randomization and assessment of IOP and tolerability were carried out at baseline, 1 month, 3 months, 4 months, and 6 months after randomization. The investigator could choose to have a nonscheduled safety visit in between the scheduled visits. At the baseline visit, patients' ophthalmic and systemic history were recorded and gonioscopy, pachymetry, and standard automated achromatic perimetry were performed. Patients then were randomized to be treated with either bimatoprost plus timolol or travoprost plus timolol, both dosed in the evening at 9 PM. A complete ophthalmologic examination, including visual acuity and refraction and lid and slit-lamp examination, was performed at the baseline, month 3, and month 6 visits. Additionally, IOP was measured at $8 \mathrm{AM}, 11 \mathrm{AM}, 2$ PM, 5 PM, and 8 PM by Goldmann applanation tonometry. Month 1 and 4 visits were safety visits; IOP was measured at $11 \mathrm{Am}$, and adverse events were recorded.

- STUDY OUTCOMES: The primary outcome was to compare the mean diurnal IOP from the daily curve after 3 months of bimatoprost plus timolol and travoprost plus timolol treatment. Secondary outcomes were to compare between groups the diurnal IOP change from baseline 3 months after the start of each treatment (for each time point separately and for the mean IOP), mean morning ( 8 AM, 11 AM), and mean afternoon IOP (2 PM, 5 PM, 8 PM), IOP change from baseline at 1 month after the start of each treatment, diurnal IOP change from baseline after switch to the other therapy, distribution of IOP reductions from baseline expressed as percentages, and tolerability.

Double masking was performed by relabeling and repackaging the study drugs in identical boxes. Each patient received after randomization an identical masked box containing 4 smaller masked boxes, each containing 1 masked bottle with the first treatment ( 1 bottle per month of treatment plus 1 extra safety bottle). At the 12-week visit (crossover visit), a second box containing 4 packed bottles of the second treatment were dispensed to each patient. Each patient eligible for the trial was allocated to 1 of the 2 treatment sequences (either first bimatoprost plus timolol for 3 months and thereafter travoprost plus timolol for 3 months or vice versa) according to a per-center computer-generated randomization code list.

- Clinical tOlerability ASSESSMENTS: Best-corrected visual acuity (Snellen chart), biomicroscopy, and ophthalmoscopy were recorded at the pretrial visit and at each follow-up visit. Any kind of adverse event was recorded. Changes of conjunctival hyperemia were recorded at the slit lamp using a standard scale ( 0 to 3 , where 0 is none, 1 is mild, 2 is moderate, and 3 is severe) with the help of a standardized photographic chart. ${ }^{16}$ Superficial keratitis, defined as the presence of small circular epithelial erosions in the cornea, was also graded as none, mild, moderate, and severe (none $=$ no staining; mild $=$ rare stained erosions localized close to the lid margins; moderate $=$ rare stained erosions localized in the 4 quadrants; diffuse $=$ diffuse stained erosions involving the 4 quadrants). 
TABLE. Mean Daily IOP (95\% Confidence Intervals) at Baseline and after 3 Months of Treatment with Travoprost and Timolol and with Bimatoprost and Timolol Fixed Combinations

\begin{tabular}{lccccc}
\hline & Baseline IOP & 3-Month IOP & Mean IOP Change & Mean $\%$ IOP Change $P$ Value \\
\hline Travoprost plus timolol & $16.5(16.0$ to 17.0$)$ & $15.4(15.0$ to 15.9$)$ & $-1.06(-0.62$ to -1.49$)$ & $-5.7 \%$ & $<.0001$ \\
Bimatoprost plus timolol & & $14.7(14.3$ to 15.3$)$ & $-1.72(-1.34$ to -2.10$)$ & $-10.1 \%$ & $<.0001$ \\
$P$ value $^{b}$ & - & .0041 & .0041 & -0023 & - \\
\hline
\end{tabular}

IOP = intraocular pressure.

aWithin-group comparison to baseline.

${ }^{b}$ Between-group comparison.

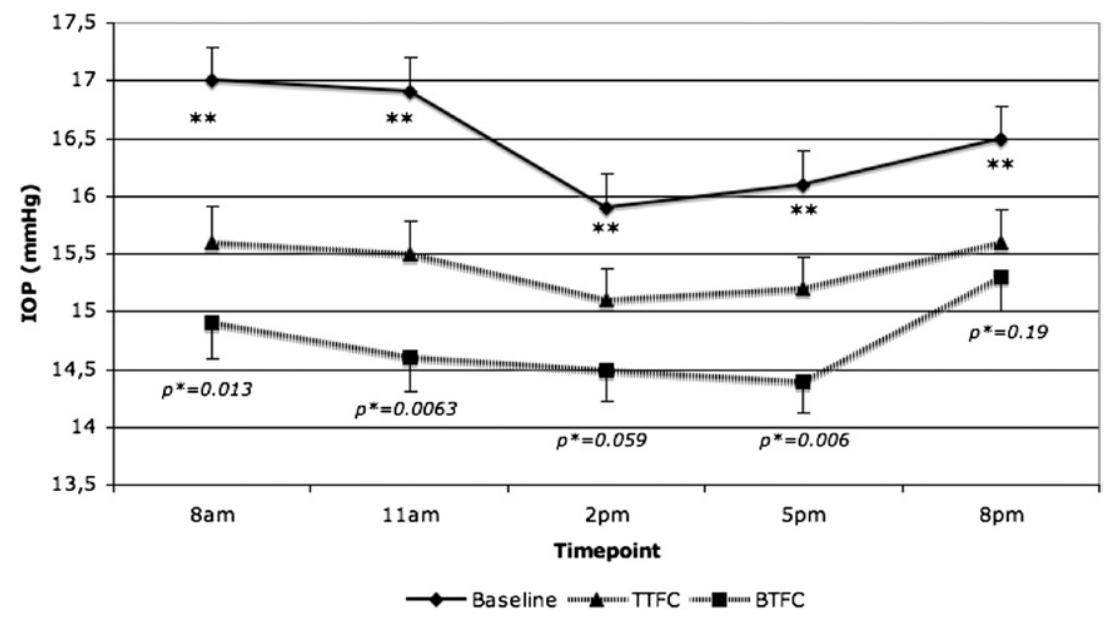

FIGURE 1. Graph showing intraocular pressure (IOP) at each time point of the daily curve at baseline and with treatment with travoprost and timolol fixed combination (TTFC) and bimatoprost and timolol fixed combination (BTFC). *Values from the comparison between drug groups of each time point of the daily curve. $* * P<0.01$ from the comparison of each drug group time point with the corresponding baseline time point.

- ANALYSIS: Data are presented as mean and 95\% confidence intervals for continuous variables and frequencies for categorical variables. For the IOP recordings, the mean value of 2 measurements at each time point (or the median of 3 readings if the first 2 were not within $2 \mathrm{~mm} \mathrm{Hg}$ ) was used in the calculations. In addition, if both eyes fulfilled the eligibility criteria, the worse eye (i.e., with higher IOP) was used in the analysis. An intent-to-treat approach was used to analyze the IOP variables, and in case of missing data, the last observation available was carried forward. A mixed-model analysis of variance model was used to compare the IOP variables between groups, including the following effects: patients as random effect, and drug and interaction between drug and treatment sequence as fixed effects (to explore potential carryover effects). Categorical variables such as proportions and tolerability variables were analyzed using the Pearson chi-square test or Fisher exact test as appropriate.

The sample size estimate was based on a test for superiority for a crossover study. Setting the $\alpha$ error to $5 \%$, 85 patients per group would enable to detect a difference of $1 \mathrm{~mm} \mathrm{Hg}$, assuming a standard deviation of $2.3 \mathrm{~mm} \mathrm{Hg}$ (based on previously published data $^{14}$ ) with a power of $80 \%$.

\section{RESULTS}

A TOTAL OF 91 PATIENTS WERE ENROLLED IN THIS STUDY, and 89 (mean age, $65.9 \pm 11.7$ years) were included in the analysis. One patient was lost to follow-up after randomization without performing any follow-up visits and was excluded from the IOP and tolerability analysis. One patient had a retinal detachment after randomization and before the first follow-up visit and was discontinued from the intervention and excluded from the IOP analysis, but included in the tolerability analysis, so the IOP analysis was performed on 89 patients who completed both phases of the study.

The Table reports the mean daily IOP at baseline and after 3 months of treatment with either travoprost plus timolol and bimatoprost plus timolol treatment. After 3 months of treatment, mean daily IOP was statistically significantly reduced compared with baseline in both 


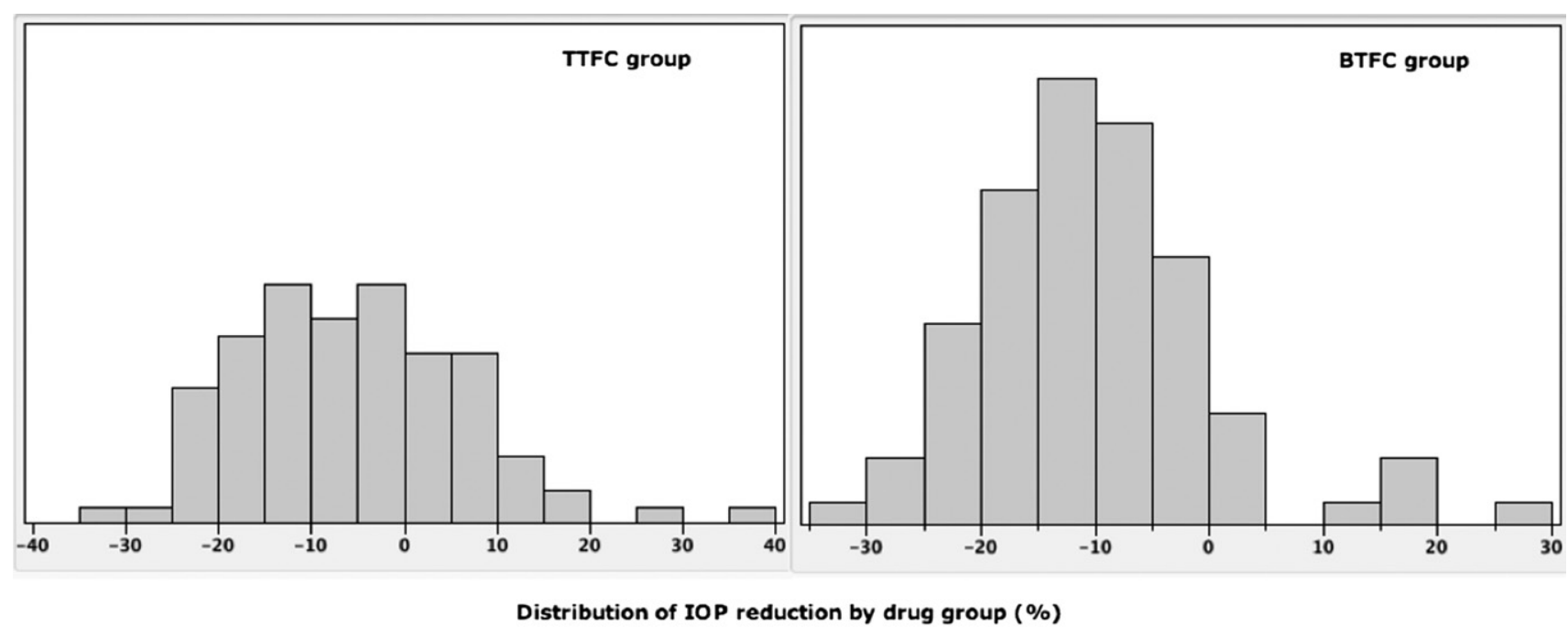

FIGURE 2. Histograms showing the distribution of percent intraocular pressure (IOP) reduction from baseline after 3 months of treatment with (Left) travoprost plus timolol fixed combination (TTFC) and (Right) bimatoprost plus timolol fixed combination (BTFC).

groups $(-1.06 \mathrm{~mm} \mathrm{Hg}, 95 \%$ confidence interval [CI], -0.62 to $-1.49 \mathrm{~mm} \mathrm{Hg}$, in the travoprost plus timolol group, $P<.001 ;-1.72 \mathrm{~mm} \mathrm{Hg}, 95 \% \mathrm{CI},-1.34$ to -2.10 $\mathrm{mm} \mathrm{Hg}$, in the bimatoprost plus timolol group, $P<.001$ ) and was statistically significantly lower in the bimatoprost plus timolol group than in the travoprost plus timolol group $(P=.0041)$. No interaction between drug and treatment sequence was detected, indicating no carry-over effects between drugs.

Mean morning IOP was statistically significantly lower in the bimatoprost plus timolol group (14.7 $\mathrm{mm} \mathrm{Hg}$; 95\% $\mathrm{CI}, 14.2$ to $15.3 \mathrm{~mm} \mathrm{Hg}$ ) than in the travoprost plus timolol group ( $15.5 \mathrm{~mm} \mathrm{Hg}$; 95\% CI, 15.0 to $16.1 \mathrm{~mm} \mathrm{Hg}$; $P=.0045)$. Mean afternoon IOP was statistically significantly lower in the bimatoprost plus timolol group (14.7 $\mathrm{mm} \mathrm{Hg}$; 95\% CI, 14.2 to $15.2 \mathrm{~mm} \mathrm{Hg}$ ) than in the travoprost plus timolol group (15.3 mm Hg; 95\% CI, 14.8 to $15.8 \mathrm{~mm} \mathrm{Hg} P=.015)$.

Figure 1 shows IOP at each individual time point of the daily curve at baseline and after 3 months of treatment with either travoprost plus timolol or bimatoprost plus timolol. IOP was lower at all time points in the bimatoprost plus timolol group, reaching statistical significance at $8 \mathrm{AM}, 11 \mathrm{AM}$, and $5 \mathrm{PM}$.

The hypotensive effect of each fixed combination after crossing over from the other fixed combination was explored, showing that mean IOP was lower during bimatoprost plus timolol treatment when used either before or after travoprost plus timolol. Baseline IOP was $16.475 \mathrm{~mm}$ $\mathrm{Hg}(95 \% \mathrm{CI}, 15.9$ to $17.0 \mathrm{~mm} \mathrm{Hg})$ in patients first treated with bimatoprost plus timolol and $16.472 \mathrm{~mm} \mathrm{Hg}(95 \%$ CI, 16.0 to $16.9 \mathrm{~mm} \mathrm{Hg}$ ) in the group first treated with travoprost plus timolol. Patients switched from travoprost plus timolol to bimatoprost plus timolol showed a mean IOP change of $-0.91 \mathrm{~mm} \mathrm{Hg}$ (from $15.46 \pm 2.38 \mathrm{~mm} \mathrm{Hg}$ to $14.55 \pm 2.78 \mathrm{~mm} \mathrm{Hg} P=.017$ ), and patients switched from bimatoprost plus timolol to travoprost plus timolol showed a mean IOP change of $+0.42 \mathrm{~mm} \mathrm{Hg}$ (from $14.94 \pm$ $1.95 \mathrm{~mm} \mathrm{Hg}$ to $15.37 \pm 2.25 \mathrm{~mm} \mathrm{Hg} ; P=.1$ ).

After 3 months of treatment, IOP was reduced from $10 \%$ to $40 \%$ from baseline in a higher proportion of patients in the bimatoprost plus timolol group compared with the travoprost plus timolol group $(54.5 \%$ vs $34.3 \%$; $P=$ .042). The histograms in Figure 2 describe the distribution of relative IOP reductions from baseline observed in each group after 3 months of treatment.

One patient allocated to travoprost plus timolol as the first treatment experienced a serious adverse event (retinal detachment) after randomization and before the first follow-up visit. The overall most commonly reported adverse event in the study was dry eye sensation, which occurred in 2 patients $(2.2 \%)$ during the bimatoprost plus timolol treatment period and in $2.2 \%$ of patients in the travoprost plus timolol treatment period. Mild punctuate keratitis was reported in those patients reporting dry eye sensation ( $2.2 \%$ of patients treated with bimatoprost plus timolol and in $2.2 \%$ of patients treated with travoprost plus timolol). Burning eye sensation occurred in 3 patients (3.4\%) receiving bimatoprost plus timolol, whereas in the travoprost plus timolol treatment period, 1 patient reported transitory blurred vision and 1 patient reported eyelash growth. Globally the incidence of adverse events was similar during both treatments. No changes in conjunctival hyperemia from baseline were reported during either treatment.

\section{DISCUSSION}

THE RESULTS OF THIS RANDOMIZED CLINICAL TRIAL SHOWED that both travoprost plus timolol and bimatoprost plus timolol provide further IOP reduction in glaucoma pa- 
tients previously treated with latanoprost plus timolol. Mean daily IOP after 3 months of treatment was statistically significantly lower during bimatoprost plus timolol treatment than during travoprost plus timolol treatment.

Assuming that the IOP-lowering effect of timolol is similar when added in fixed combination with either travoprost or bimatoprost, the findings of the present study are consistent with the results of previously published reports in which the hypotensive effect of prostaglandin analogs was compared. ${ }^{13}$ Interestingly enough, it emerged that, despite the relatively low baseline IOPs (mean, 16.5 mm Hg [95\% CI, 16.0 to 17.0 $\mathrm{mm} \mathrm{Hg]),} \mathrm{with} \mathrm{latanoprost} \mathrm{plus} \mathrm{timolol} \mathrm{treatment,} \mathrm{both}$ travoprost plus timolol and bimatoprost plus timolol were able to provide further IOP reduction after 3 months of treatment $(-1.06 \mathrm{~mm} \mathrm{Hg}$ [95\% CI, -0.62 to $-1.49 \mathrm{~mm} \mathrm{Hg}]$ and $-1.72 \mathrm{~mm} \mathrm{Hg}[95 \% \mathrm{CI},-1.34$ to $-2.10 \mathrm{~mm} \mathrm{Hg}$, respectively). These results are in agreement with recently published studies comparing bimatoprost plus timolol and latanoprost plus timolol. ${ }^{14,15}$ Centofanti and associates reported in a prospective, parallel, randomized, single-masked clinical study that bimatoprost plus timolol shows greater IOP-lowering effect compared with latanoprost plus timolol in patients previously treated with prostanoid monotherapy. ${ }^{14}$ Similarly, the study by Martinez and Sanchez suggests that bimatoprost plus timolol provides better IOP control than that of latanoprost plus timolol in primary open-angle glaucoma and pseudoexfoliative glaucoma over a 12-hour period in patients previously treated with timolol monotherapy. ${ }^{15}$

These data are also confirmed by the analysis of mean morning IOP (average of $8 \mathrm{AM}$ and $11 \mathrm{AM}$ time points) and mean afternoon IOP (average of 2 PM, 5 PM, and 8 PM time points). When looking at each individual time point of the daily curve, bimatoprost plus timolol provided consistently lower IOPs compared with travoprost plus timolol, with statistical significance reached at 3 of 5 time points of the daily curve ( $8 \mathrm{AM}, 11 \mathrm{AM}$, and $5 \mathrm{PM}$ ).

One of the advantages of prostaglandin plus timolol fixed combinations is represented by the simplified dosing regimen of 1 single drop daily compared with the more complex dosage regimen of the same compounds given concomitantly, with reduced daily exposure to topical preservatives, and potential positive effects both on medication side effects and compliance. ${ }^{18}$ When using prostaglandin plus timolol fixed combinations in glaucoma, the diurnal IOP-lowering effect may differ according to the time of instillation. ${ }^{17}$ In our study, all fixed combinations (latanoprost plus timolol before baseline and travoprost plus timolol and bimatoprost plus timolol during followup) were administered at night. Recently, it was observed by Konstas and associates that both the morning and the evening instillation are effective in reducing the 24-hour IOP and that the evening dosing gives rise to a statistically better 24-hour IOP control. ${ }^{17}$ Although statistically significant, the difference is likely not to be clinically meaningful for the small magnitude, and dosing can be adjusted to the untreated 24-hour pressure profile and the preference of the individual patient.

At month 3, the mean difference in IOP reduction from baseline between bimatoprost plus timolol and travoprost plus timolol was $-0.66 \mathrm{~mm} \mathrm{Hg}(95 \% \mathrm{CI},-0.1$ to -1.2 $\mathrm{mm} \mathrm{Hg}$ ), and it is interestingly to note that a higher proportion of patients showed relative IOP reductions between $-10 \%$ and $-40 \%$ from baseline when treated with bimatoprost plus timolol rather than with travoprost plus timolol $(54.5 \%$ vs $33.4 \%)$.

The finding of greater IOP-lowering effect of bimatoprost plus timolol also is supported by the observation of consistently lower IOPs observed during bimatoprost plus timolol treatment regardless of the treatment sequence (either before or after travoprost plus timolol in the crossover design), even if statistical significance was reached only when bimatoprost plus timolol was used after travoprost plus timolol. The lack of statistical significance could be explained by the smaller difference of mean IOP between groups even if a high probability of type II error may not be excluded for the smaller sample size of the subgroup analysis.

A similarly good tolerability profile has been shown for both fixed combinations. No changes of ocular hyperemia from baseline were reported with either treatment. This finding may be explained considering that patients at baseline were already exposed to topical prostaglandin analogs; however, it confirms other reports indicating that fixed combinations in general seem to be tolerated better than corresponding unfixed drugs. ${ }^{19}$

The lack of a washout between treatment periods may be considered as a limitation of the present study. Nevertheless, the hypotensive effect was evaluated in the present study 12 weeks after randomization and 12 weeks after crossing over, when any previous treatment was likely to be completely washed out.

In conclusion, this study suggests that travoprost plus timolol and bimatoprost plus timolol both given once daily in the evening effectively reduces the IOP in patients not well controlled with latanoprost plus timolol. Moreover, bimatoprost plus timolol has shown a greater IOP reduction compared with travoprost plus timolol with similar tolerability and tolerability profile. Although the results of this study seem to be promising, the clinical relevance needs to be determined in further studies considering also that a regression to the mean could account for some of the effects noted. The results of previously published large, randomized clinical trials reported that every $1 \mathrm{~mm} \mathrm{Hg}$ of lower IOP obtained by treatment is associated with a reduced risk of disease progression, ${ }^{2,4}$ and it would be interesting to investigate if the differences in IOP observed in the present study can have clinical relevance in influencing the rate of progression of the disease and ultimately the long-term individual visual function prognosis. 
PUBLICATION OF THIS STUDY WAS SUPPORTED BY AN UNRESTRICTED GRANT FROM ALLERGAN LTD., BUCKINGAMSHIRE, United Kingdom. Dr Centofanti has received lecture fees from Alcon, Allergan, Pfizer, and MSD grant support from Allergan and MSD. Dr Oddone has received consulting fees from Allergan, MSD, Pfizer, and Omikron Italia. Dr Gandolfi has received consulting fees from Alcon and Allergan; lecture fees from Alcon and MSD; and grant support from Regione Emilia Romagna and Regione Puglia. Dr Hommer has received consulting fees from Allergan, Pfizer, and MSD and lecture fees from Alcon, Allergan, MSD, Pfizer, and Santen. Dr Boehm has received lecture fees from Allergan and Pfizer. Dr Manni has received consulting fees from Alcon, Allergan, and MSD and grant support from Allergan and MSD. Dr Rossetti has received lecture fees from Alcon, Allergan, Pfizer, and MSD and grant support from Alcon, Allergan, Pfizer, MSD. The remaining authors indicate no financial conflict of interest. Involved in conception and design (M.C., F.O., L.R.); Analysis and interpretation of data (M.C., F.O., G.M., L.R.); Writing article (M.C., F.O.); Critical revision of article (M.C., S.G., A.H., A.B., G.M., L.R.); Data collection (C.S., L.T., V.S., M.H.); Provision of materials, patients, or resources (M.C., S.G., A.B., L.R., A.H.); Statistical expertise (F.O., L.R.); Obtaining funding (M.C., L.R.); Literature search (F.O., L.T.); Administrative or logistic support (F.O., L.T., C.S., V.S., M.H.); and Final approval (M.C., F.O., L.R.A.H., A.B., G.M., L.R.). The study was conducted in accordance with the ethical principles that have their origins in the Declaration of Helsinki and its amendment of October 2000 (Edinburgh, United Kingdom), ethics committee approval was obtained from the Institutional Review Board of each center, and informed consent for the research was obtained from each participating patient. The study was registered in the European clinical trial database with number Eudract 2007-004023-38.

\section{REFERENCES}

1. Maier PC, Funk J, Schwarzer G, Antes G, Falck-Ytter YT. Treatment of ocular hypertension and open angle glaucoma: meta-analysis of randomised controlled trials. BMJ 2005; 331(7509):134-139.

2. Leske MC, Heijl A, Hussein, et al. Factors for glaucoma progression and the effect of treatment: the Early Manifest Glaucoma Trial. Arch Ophthalmol 2003;121(1):48-56.

3. The AGIS Investigators. The Advanced Glaucoma Intervention Study (AGIS): 7. The relationship between control of intraocular pressure and visual field deterioration. Am J Ophthalmol 2000;130(4):429-440.

4. Chauhan BC, Mikelberg FS, Balaszi AG, et al. Canadian Glaucoma Study. Arch Ophthalmol 2008;126(8):1030-1036.

5. European Glaucoma Society (EGS). Terminology and Guidelines for Glaucoma. Editrice Dogma s.r.1., Savona, Italy, 2008: $1-183$.

6. Alm A. Prostaglandin derivates as ocular hypotensive agents. Prog Retin Eye Res 1998;17(3):291-312.

7. Alexander CL, Miller SJ, Abel SR. Prostaglandin analog treatment of glaucoma and ocular hypertension. Ann Pharmacother 2002;36(3):504-511.

8. Hoyng PF, Van Beek Lm. Pharmacological therapy for glaucoma: a review. Drugs 2000;59(3):411-434.

9. Kass MA, Heuer DK, Higginbotham EJ, et al. The Ocular Hypertension Treatment study. A randomized trial determines that topical ocular hypotensive medication delays or prevents the onset of primary open angle glaucoma. Arch Ophthalmol 2002;120(6):701-713.

10. Lichter PR, Musch DC, Gillespie BW, et al. Interim clinical outcomes in the Collaborative Initial Glaucoma Treatment Study comparing initial treatment randomized to medication or surgery. Ophthalmology 2001;108(11):1943-1953.
11. Chrai SS, Makoid MC, Eriksen SP, Robinson JR. Drop size and initial dosing frequency problems of topically applied ophthalmic drugs. J Pharm Sci 1974;63(3):333-338.

12. Dunker Sm, Schmucker A, Maier H. Tolerability, quality of life, and persistency of use in patients with glaucoma who are switched to the fixed combination of latanoprost and timolol. Adv Ther 2007;24(2):376-386.

13. Aptel F, Cucherat M, Denis P. Efficacy and tolerability of prostaglandin analogs: a meta-analysis of randomized controlled clinical trials. J Glaucoma. 2008;17(8):667-673.

14. Centofanti M, Oddone F, Vetrugno M, et al. Efficacy of the fixed combinations of bimatoprost or latanoprost plus timolol in patients uncontrolled with prostaglandin monotherapy: a multicenter, randomized, investigator-masked, clinical study. Eur J Ophthalmol 2009;19(1):66-71.

15. Martinez A, Sanchez M. Bimatoprost/timolol fixed combination vs latanoprost/timolol fixed combination in open-angle glaucoma patients. Eye 2009;23(4):810-818.

16. Cornea and Contact Lens Research Unit (CCLRU) Grading Scales (1996). University of New South Wales, School of Optometry, Sydney, Australia.

17. Konstas AG, Hollo G, Mikropoulos D, et al. Twenty-four hour intraocular pressure control with bimatoprost and bimatoprost/timolol fixed combination administered in the morning, or evening in exfoliative glaucoma. $\mathrm{Br} \mathrm{J}$ Ophthalmol 2010;94(2):209-213.

18. Dunker S, Schmucker A, Maier H. Tolerability, quality of life, and persistency of use in patients with glaucoma who are switched to the fixed combination of latanoprost and timolol. Adv Ther 2007;24(2):376-386.

19. Khouri AS, Realini T, Fechtner RD. Use of fixed-dose combination drugs for the treatment of glaucoma. Drugs Aging 2007;24(12):1007-1016. 